\title{
Transport, Targeting, and Applications of Metallic Functional Nanoparticles for Degradation of DNAPL C hlorinated Organic solvents
}

\author{
EMSP P roject Number 86981
}

George Redden, Idaho National Engineering and Environmental Laboratory G regory V. Lowry, Carnegie Mellon University

Dan G inosar, National Engineering and Environmental Laboratory

Sara Majetich, Carnegie Mellon University

Krzysztof Matyjaszewski, Carnegie Mellon University

Paul Meakin, National Engineering and Environmental Laboratory

Harry Rollins, National Engineering and Environmental Laboratory

David Sholl, Carnegie Mellon University

Robert Tilton, Carnegie Mellon University

Number or graduate students involved in project:

2-graduate students

\section{Project 0 bjectives}

This project addresses the need for methods to remove or degrade subsurface contaminants that are present as dense non-aqueous phase liquids (DNAPLs), and act as long-term sources of groundwater contamination. The goal is to build on a particle-based approach to subsurface contaminant remediation that is based partly on the recent success in using nanoparticle iron to degrade chlorinated compounds dissolved in groundwater, and knowledge of how colloids migrate in porous media. The objective is to engineer reactive nanoparticles that can decompose and potentially isolate DNAPL pollutants in the subsurface. Delivering reactive particles directly to the surface of the DNAPL will decompose the pollutant into benign materials, reduce the migration of pollutant during treatment, possibly lead to encapsulation of the DNAPL, and reduce the time needed to remove residual pollution by other means, such as natural attenuation.

Contributions from several basic science fields will be needed to advance a particle-based strategy for in situ DNAPL degradation by providing targeted delivery of reactive particles directly to the DNAPL.

1. Design and synthesize reactive $\mathrm{Fe}(0)+$ +catalyst particles that have surfaces modified with organic polymers such that the particles partition to/in DNAPLS.

2. Determine how variables such as size, composition, availability of water, and exposure to the transporting medium affect their ability to degrade TCE.

3. Investigate the transportability and water-DNAPL partitioning of particles in saturated porous media using micro-model and intermediate scale experiments in porous media. Determine how physical, chemical, and 
hydrologic regimes existing in the subsurface affect nanoparticle migration.

4. Test a method of targeted delivery of particles to DNAPLs particle suspensions in a benign organic medium with properties engineered to reflect those that govern DNAPL migration.

5. Develop a predictive numerical model for the transport and degradation of DNAPLs in bench-scale and meso-scale experiments. 


\section{Research Progress and Implications}

This project was initiated September 15, 2002. Research has focused on numbers 1 ) determining the effects of particle composition and history on the ability to degrade TCE, 2) Designing and synthesizing inorganic-organic hybrid particles with the appropriate water solubility and TCE partitioning behavior, and 5) developing the numerical models to predict transport and partitioning behavior of the nanoparticles.

1) Designing and synthesizing inorganic-organic hybrid particles with the appropriate water solubility and TCE partitioning behavior. - Atom Transfer Radical Polymerization (ATRP) was used to design tailored block copolymers starting from easily available raw materials. The hybrid nanoparticles have an inorganic core with a block copolymer shell (hydrophobic inner shell surrounded with a hydrophilic outer shell). The hydrophobic inner shell protects the zerovalent iron from the contact with water before reaching the DNAPL, and the hydrophilic shell enables transport of the particle in water to the contaminated areas. Characterization studies have been conducted on unsupported block copolymers to determine the nature and length of the hydrophobic and hydrophilic blocks, and identify good candidates for use in transport/degradation experiments. Silica supported block copolymers have been developed for use in transport experiments, and iron supported nanoparticles have been developed for use in transport/TCE degradation experiments.

- Polymer Blocks. Poly(styrene) and poly(methyl methacrylate) have been identified as good candidates for the hydrophobic blocks. Sulfonated polystyrene has excellent water solubility and makes a good hydrophilic block. Poly(ethylene oxide) methacrylate segments strongly adhered to silica due to the poly(ethylene oxide) side chains. This is undesired because it will limit the transportability of the hybrid nanoparticles in the subsurface. These copolymers have been excluded from the future studies although they also strongly adhere to iron particles.

- Silica core-shell inorganic-organic hybrid nanoparticles. A method was developed to synthesize silica-supported initiators starting from $20 \mathrm{~nm}$ silica dispersed in methyl ethyl ketone. Polymerizing styrene using the silica-immobilized initiators provided nanoparticles with attached polystyrene chains $(\mathrm{d} \sim 120 \mathrm{~nm})$. After sulfonation the particles $(\sim 90 \mathrm{~nm})$ were completely soluble in water.

- $\mathrm{Fe}(0)$ core-shell inorganic-organic hybrid nanoparticles. A novel strategy was developed to attach polymeric chains to iron nanoparticles. Triblock copolymers were attached to $\mathrm{Fe}(0)$ particles. The first block was a poly(methacrylic acid) segment capable of binding to the $\mathrm{Fe}(0)$ particle. The second block was hydrophobic poly(methyl methacrylate). The third block was polystyrene which after sulfonation, should provide good water solubility and stability. The prepared iron hybrid nanoparticles had bimodal particle size distribution, indicating some aggregation. However, after the temperature of the reaction mixture was cycled (warmed to $50{ }^{\circ} \mathrm{C}$ then 
cooled down to RT) a monomodal distribution was observed, indicating the absence of any aggregation.

Implications. Appropriate hydrophobic and hydrophilic polymer blocks were identified and the first generation of inorganic-organic hybrid nanoparticles is now available for characterization and transport experiments. Characterization/testing of these particles will provide feedback to improve the next generation of particles. The ability to functionalize $\mathrm{SiO}_{2}$ and $\mathrm{Fe}(0)$ particles has been demonstrated.

Future work. The ratios of hydrophilic/hydrophobic blocks will be varied to better control the water solubility, transport, and particle protection provided by the hydrophobic block. The poly (acrylic acid block size will be varied to optimize polymer attachment to iron particles.

2) Determining the effects of particle composition and history on the ability to degrade TCE : Iron nanoparticle reactivity will eventually be limited by the thickness of the oxide shell that forms around the particle, but it is unknown what that thickness may be, or the factors controlling the rate and phase of oxide growth. We have begun characterizing and evaluating the reactivity/lifetime of two types of iron particles, commercially produced needle-shaped $200 \mathrm{~nm}$ by 20 $\mathrm{nm}$ particles from Toda, Inc., and 1 micron aggregates of $100 \mathrm{~nm}$ spherical iron particles made from borohydrate reduction of $\mathrm{Fe}^{2+}$ in aqueous solution.-Key findings thus far are:

- Iron particles made from the borohydrate reduction, even those that are stabilized in air, are highly reactive. Air stabilized particles initially dechlorinate TCE at a slower rate, but later dechlorinate TCE at a similar rate as freshly reduced particles. Freshly reduced iron particles from Toda, Inc., which contain no boron, are not as reactive, even though they contain more $\mathrm{Fe}(0)$ than particles made from borohydrate reduction.

- Electron diffraction of both types of particles reveals only the bcc structure of $\alpha-\mathrm{Fe}(0)$. However, the saturation magnetization of fully reduced borohydrate particles $(\sim 120 \mathrm{emu} / \mathrm{g})$ is lower than that of magnetic the Toda Inc. particles ( $200 \mathrm{emu} / \mathrm{g})$ or pure $\alpha-\mathrm{Fe}(0)(\sim 215 \mathrm{emu} / \mathrm{g})$.

- Only trace amounts of chlorinated intermediates are produced during TCE degradation.

Implications. Particles made form borohydrate reduction of $\mathrm{Fe}^{2+}$ in water have several favorable properties relative to TCE degradation. Their ability to be stabilized in air and retain their activity makes handling and subsurface delivery easier. The borohydrate particles have a significant amount of either interstitial boron or a highly disordered surface phase that plays a key role in their reactivity. The role of the boron could be to reduce the amount of iron oxide formed. Iron oxide would be less electronically conductive, and therefore a bigger barrier to electron transfer needed to reduce TCE.

Future work. Full characterization of each type of particle will be performed. Particle properties will be correlated with their ability to dechlorinate TCE to optimize the properties of the iron nanoparticles. 
3) Investigate the transportability and water-DNAPL partitioning of particles in saturated porous media using micro-model and intermediate scale experiments in porous media. Determine how physical, chemical, and hydrologic regimes existing in the subsurface affect nanoparticle migration: This phase of the project has not yet been initiated.

4) Test a method of targeted delivery of particles to DNAPLs particle suspensions in a benign organic medium with properties engineered to reflect those that govern DNAPL migration. This phase of the project has not yet been initiated.

5) Developing the numerical models to predict transport and partitioning behavior of the nanoparticles. - The diffusion rates of nanoscale particles, particularly in inhomogeneous environments such as water/DNAPL interfaces, are sensitive to the microscopic particle structure. Their structure is challenging to fully characterize experimentally, so computer simulations on molecular lengthscales (Brownian Dynamics (BD) simulations) are being conducted to describe the dynamics and structure of polymer-grafted metal nanoparticles. These simulations will complement experimental particle characterization and, ultimately, will provide parametric inputs for macroscale simulations of particle transport in porous media.

- BD simulations of homopolymers. The dynamics of homopolymers in solution were modeled using a bead-spring model with a Padé approximation for the Inverse Langevin function. Simulation results for the structure and dynamics of polystyrene in a theta solvent during extensional flow with prior $\mathrm{BD}$ simulations ${ }^{1}$ yielded excellent agreement between the two calculations.

- BD simulations of nanoparticles. For our purposes, simulating the Brownian Dynamics of a solid nanoparticle in a solvent is somewhat more challenging than simply reproducing the known translational diffusivity of solid particles, since we aim to correctly describe the dynamics of polymers grafted to the particle. As a result, the description of the solid particle must reproduce the correct translational and rotational diffusivities. Furthermore, the rigid nature of the particle must be included in the simulation. These tasks were accomplished by performing BD simulations of a set of nodes distributed on the surface of the nanoparticle subject to distance constraints ${ }^{2}$. A Monte Carlo algorithm is used to define the positions of these nodes on the nanoparticles surface in a way that approximately maximizes the distances between nodes. This definition of the node position is a natural one for describing sites to which polymers can be grafted on the particle. An additional set of nodes not sitting on the true surface of the particle are also included in the description of the particle in order to give the composite set of nodes the same moment of inertia as the true rigid nanoparticle. With this multi-site description, BD simulations of the nanoparticle (with no grafted polymer present) exhibit 
the correct translational and rotational diffusivities for a solid particle in a homogeneous solvent.

- BD simulations of inorganic-organic hybrid nanoparticles. Bead-spring models of homopolymers grafted to solid nanoparticles have been converted to forms suitable for use in BD simulations of inorganic-organic hybrid nanoparticles, and preliminary simulations are being performed for solid nanoparticles with short homopolymer chains grafted to them. Implications. Measurements of the polymer structure and the translational diffusivity of the composite particle are providing information that can be closely related to the experimental data available from light scattering. Working in tandem with light scattering experiments, we expect to be able to develop our BD simulations to a level where they can provide quantitative information supporting choices of graft densities, polymer chain lengths, etc. within the overall context of this project.

Future work. Hydrodynamic interactions will be included in the BD simulations so that we can compare model results with experiments using silica and iron particles with sulfonated polystyrene. The sulfonated polystyrene is charged and this can affect the dynamic light scattering results. Particle-silica and particleorganic interactions will then be incorporated into the model.

${ }^{1}$ L. Li, R.G. Larson and T. Sridhar, J . Rheol., 44, p. 291 (2000)

2 J. Ryckaert, G. Ciccotti, and H. J . C. Berendsen, J . Computational Physics, 23, p. 327-341 (1977) 